\title{
Individual differences in spatial configuration learning predict the occurrence of intrusive memories
}

\author{
Thomas Meyer • Tom Smeets • Timo Giesbrecht • \\ Conny W. E. M. Quaedflieg • Marta M. Girardelli • \\ Georgina R. N. Mackay • Harald Merckelbach
}

Published online: 23 September 2012

(C) Psychonomic Society, Inc. 2012

\begin{abstract}
The dual-representation model of posttraumatic stress disorder (PTSD; Brewin, Gregory, Lipton, \& Burgess, Psychological Review, 117, 210-232 2010) argues that intrusions occur when people fail to construct context-based representations during adverse experiences. The present study tested a specific prediction flowing from this model. In particular, we investigated whether the efficiency of temporal-lobe-based spatial configuration learning would account for individual differences in intrusive experiences and physiological reactivity in the laboratory. Participants $(N=82)$ completed the contextual cuing paradigm, which assesses spatial configuration learning that is believed to depend on associative encoding in the parahippocampus. They were then shown a trauma film. Afterward, startle responses were quantified during presentation of trauma reminder pictures versus unrelated neutral and emotional pictures. PTSD symptoms were recorded in the week following participation. Better configuration learning performance was associated with fewer perceptual intrusions, $r=-.33, p<.01$, but was unrelated to physiological responses to trauma reminder images ( $p$ s > .46) and had no direct effect on intrusion-related distress and overall PTSD symptoms, $r \mathrm{~s}>-.12, p \mathrm{~s}>.29$. However, configuration learning performance tended to be associated with reduced physiological responses to unrelated negative images, $r=-.20, p=.07$. Thus, while spatial configuration learning appears to be unrelated to affective responding to trauma reminders, our overall findings support the idea that the context-based memory system helps to reduce intrusions.
\end{abstract}

Electronic supplementary material The online version of this article (doi:10.3758/s13415-012-0123-9) contains supplementary material, which is available to authorized users.

T. Meyer $(\bowtie) \cdot$ T. Smeets $\cdot$ T. Giesbrecht $\cdot$

C. W. E. M. Quaedflieg • M. M. Girardelli - G. R. N. Mackay •

H. Merckelbach

Faculty of Psychology and Neuroscience, Maastricht University, P.O. Box 616, 6200 MD Maastricht, The Netherlands

e-mail: thomas.meyer@maastrichtuniversity.nl
Keywords Posttraumatic stress disorder · Intrusions · Spatial contextual cuing task $\cdot$ Startle paradigm

Many people are exposed to potentially traumatic events, such as life-threatening accidents, being held captive, or the death of a close friend, at some point in their life. A possible negative outcome of the fear, horror, or helplessness that may accompany these events is posttraumatic stress disorder (PTSD). Trauma victims who develop PTSD suffer from prolonged reactions to the event, including reexperiencing (e.g., intrusions, nightmares), avoidance of cues and situations related to the trauma, emotional numbing, and increased general arousal (American Psychiatric Association, 2000). Surprisingly, only a minority of those who have been exposed to potentially traumatic experiences actually do develop PTSD. Indeed, most people are able to adapt well to adverse experiences, a phenomenon called resilience (Bonanno, 2004). Researchers and clinicians alike have been intrigued by the question of what distinguishes people who experience pathological symptoms after adversity from those who are resilient.

Studies of the predictive factors of PTSD strongly suggest that resilience is related to individual differences in biopsychological reactions during and shortly after the traumatic event (e.g., Marmar et al., 2006; for a meta-analysis, see Ozer, Best, Lipsey, \& Weiss, 2003). For instance, alterations in physiological and hormonal activity known to influence memory have been shown to be predictive of later PTSD (Delahanty \& Nugent, 2006). Accordingly, a prominent theoretical account of PTSD (e.g., de Quervain, Aerni, Schelling, \& Roozendaal, 2009; Elzinga \& Bremner, 2002) posits that physiological hyperactivity and hormonal deregulation during a traumatic experience lead to dysfunctional activity in memory-encoding structures of the brain-notably, the amygdala and hippocampus - which could lead to the development of PTSD. 
The dual-representation model by Brewin and colleagues (Brewin et al. 2010) is a detailed neuroanatomical model of memory formation during traumatic experiences aiming to account for the above-mentioned findings (for competing or complementing models, see, e.g., Francati, Vermetten, \& Bremner, 2007; Rubin, Berntsen, \& Bohni, 2008; Shin, Rauch, \& Pitman, 2006; Suvak \& Barrett, 2011). According to this model, two distinct but interacting memory systems in the brain are responsible for the occurrence of intrusive memories, a hallmark symptom of PTSD. The first system is a sensation-based memory system, which would encode perceptual and affective qualities of events in cortical and subcortical sensory areas, including the insula and the amygdala. The processing of information within this system purportedly is enhanced during stressful situations. The second system is a context-based memory system that would support further processing of the sensory input both by recruiting sensory association areas and by translating the egocentric viewpoint of the sensory input into abstract, allocentric representations. The brain structures involved in context-based memory are the hippocampus and parahippocampus.

According to the dual-representation model (Brewin et al., 2010), intrusions occur when highly emotional sensation-based memories are formed in the absence of a complete context-based memory for the traumatic event. When reactivated, a sensation-based memory will therefore be experienced from an egocentric viewpoint, such that the reactivated affective states are perceived as having immediate significance. Thus, according to Brewin et al., the formation of allocentric, context-based memory prevents or at least reduces the occurrence of intrusions. Therefore, one would expect variability in the efficiency with which contextual representations are encoded to underlie individual differences in resilience to adversity.

To date, the most direct evidence in support of the dualrepresentation model comes from two studies that assessed individual differences in visuospatial processing abilities and related them to PTSD symptoms (Bisby, King, Brewin, Burgess, \& Curran, 2010; Gilbertson et al., 2007). In an elegant twin study, Gilbertson et al. showed that PTSD was marked by impaired allocentric visuospatial processing, as measured with a 20-item multiple-choice task in which participants had to rotate cubes in their minds or visualize how papers would be folded. More recently, Bisby et al. examined the occurrence of intrusions in a trauma film paradigm using a spatial memory task in which participants explicitly memorized spatial locations in a 3-D virtual space. Spatial learning was followed by recognition trials either from the same spatial viewpoint or from a shifted viewpoint, whereby the latter trials necessarily required a viewpointindependent (i.e., allocentric) memory representation. Bisby and colleagues found that better recognition performance from a shifted viewpoint correlated with fewer perceptual intrusions. Thus, the aforementioned studies suggest that allocentric spatial processing and recognition may play an important role in intrusive memories. In line with the predictions of the dual-representation model (Brewin et al., 2010), these findings suggest that allocentric visuospatial processing and memory formation could be associated with individuals' ability to form contextualized memories of a traumatic experience.

The tasks used in both studies (Bisby et al., 2010; Gilbertson et al., 2007) required complex mental operations (e.g., explicit memorizing, mental orientation, visualization) that likely tapped the efficiency of the hippocampus (see Burgess, Maguire, \& O'Keefe, 2002), as well as structures in the parietal cortex (Jordan, Heinze, Lutz, Kanowski, \& Jancke, 2001). Importantly, the dual-representation model ascribes important roles to both the hippocampal area (e.g., the formation of contextbased memories) and parietal regions in the development of intrusions (e.g., promoting the interaction between sensationbased and context-based memories; Brewin et al., 2010). Thus, the more specific involvement of a hippocampal-area-based memory system in the development of intrusions, as hypothesized in the dual-representation model, remains to be tested empirically.

To explore the specific link between hippocampal-areabased memory formation and intrusive experiences, we used the contextual cuing paradigm (Chun \& Jian, 1998) to assess individual differences in the efficiency with which visuospatial context information is encoded. This paradigm measures the degree to which spatial configurations of multiple simple cues are bound in memory. Although this learning performance is not necessarily allocentric in nature (e.g., Chua \& Chun, 2003), studies have suggested that contextual cuing crucially depends on structures in the parahippocampus (Chun \& Phelps, 1999; Manns \& Squire, 2001; Preston $\&$ Gabrieli, 2008) that are involved in the encoding of associative feature conjunctions (Fyhn, Molden, Witter, Moser, \& Moser, 2004; Murray, Bussey, \& Saksida, 2007; van Strien, Cappaert, \& Witter, 2009) and serve as major input for the construction of spatial representations in the hippocampus (Fyhn, Hafting, Treves, Moser, \& Moser, 2007). Therefore, reduced learning efficiency on the contextual cuing task might reflect a reduction of information processing that would be a major input for a coherent hippocampal-dependent representation during a traumatic event. Thus, the contextual cuing paradigm can be used to provide additional insights into the relationship between contextual memory formation in the medial temporal lobe and intrusive memories of highly emotional events.

Aside from assessing the efficiency of visuospatial context-based memory formation, we subjected participants to a trauma film paradigm (Holmes, Brewin, \& Hennessy, 2004) and measured auditory startle responses $30 \mathrm{~min}$ later 
to assess affective responding (Jackson et al., 2003) to reminder pictures. Importantly, startle probes were presented both during and after picture presentation, making it possible to independently assess affective responding and affective down-regulation, respectively. Reminder-related affective responding may be especially relevant for individual differences in resilience, since PTSD patients, in contrast to resilient individuals, often show a specific exaggeration of startle responses toward trauma-related stimuli (Orr \& Roth, 2000). Heightened startle responses to fear-associated stimuli is indicative of amygdala activation (Davis, Walker, \& Lee, 1997), which is thought to be central in the processing of sensation-based memories (Brewin et al., 2010). Thus, in terms of the dual-representation model, the startle responses during memory activation may be indicative of the strength of film-related affective memories. By contrast, affective down-regulation of startle responses is essentially thought to be mediated by the frontal cortex (Jackson et al., 2003) under guidance of contextual memory (Thayer, Ahs, Fredrikson, Sollers, \& Wager, 2012). Although the affective reaction to trauma memories may depend on a number of other mechanisms (e.g., selective attention, negative appraisals, avoidance, cognitive distortions; Brewin et al., 2010), the dual-representation model would predict that the efficiency of forming contextual representations of the trauma film contributes to affective down-regulation of startle responses. Finally, the occurrence of PTSD symptoms induced by the distressing film fragments was assessed using a 1-week intrusion diary and self-report measures of PTSD symptoms related to the trauma film.

On the basis of these considerations, we hypothesized that the efficiency of visuospatial context-based memory formation would be related to fewer intrusions in the week following exposure to the distressing film fragments. Additionally, we expected that this efficiency would lead to enhanced affect regulation in response to the activation of trauma memories as indexed by modulation of responses in the auditory startle paradigm. Concerning self-reported PTSD symptoms, more efficient context-based memory formation was expected to predict fewer PTSD symptoms in general (in terms of overall scores on PTSD scales) and fewer intrusions and lower intrusion-related distress in particular.

\section{Method}

Participants

Eighty-two undergraduates (63 women) with a mean age of 21.5 years $(S D=3.4)$ completed the study. Participants were recruited via advertisements at the university campus and received partial course credit or a small financial compensation in return for their participation. Exclusion criteria (based on self-report) were (1) recent psychological complaints, (2) drug or alcohol abuse or addiction, (3) blood phobia, and (4) past traumatic experiences that were similar to those depicted in the used trauma film fragments (see the Procedure section below; i.e., serious car accidents, lifethreatening injuries, serious violence). Participants were informed that the materials used in the study might cause transient negative emotions and intrusions. In a follow-up inquiry, none of the participants reported long-term distress from the study. All participants had normal or corrected-tonormal vision. This study was approved by the standing ethical committee of the Faculty of Psychology and Neuroscience, Maastricht University. All participants provided written informed consent.

\section{Materials}

\section{Spatial contextual cuing task}

The contextual cuing paradigm (Chun \& Jian, 1998) requires participants to find a single target among a number of distractor stimuli. On half of the trials, the configuration of the distractor stimuli (i.e., the visuospatial context of the target) is repeated, while on the other half of the trials, new distractor configurations are presented. The repeated target contexts predict the location of the target and, thereby, facilitate the search, as evidenced by shorter reaction times (RTs) in comparison with new distractor configurations. This RT difference is an index of the contextual learning effect (Chun \& Jian, 1998). We used the abbreviated spatial contextual cuing task (SCCT) developed by Bennett and colleagues (Bennett, Barnes, Howard, \& Howard, 2009). Prior to the task, participants were given a passive strategy instruction in order to decrease performance variation due to different cognitive search strategies (see Lleras \& Von Muhlenen, 2004). Specifically, participants were asked to use their intuition rather than systematic search to find the target. The task consisted of 30 blocks containing six trials with repeated arrays and six trials with new arrays (for more details about stimuli and task construction, see Text 1, Supplementary Materials). RT data were condensed by averaging the median RTs across three epochs of 10 blocks. Similar to prior studies (e.g., Bennett et al., 2009), scores of configuration learning were calculated for each epoch by subtracting the score for repeated arrays from the score for novel arrays per epoch. Also, average accuracy scores were calculated for each epoch and array type.

We found the typical contextual cuing effect in the present sample (i.e., shorter RTs in repeated arrays, as compared with novel arrays; for details, see Text 2, Supplementary Materials). We also observed strong nonsphericity in the array $\times$ epoch interaction, because participants with better initial configuration learning reached a ceiling of the 
learning effect already in the first epoch (see Text 2 and Fig. 1, Supplementary Materials). Therefore, the average median RT difference in the first epoch $(M=96.4 \mathrm{~ms}$, $S D=411.4 \mathrm{~ms}$ ) most likely reflects individual learning differences and was used to index encoding efficiency (hereafter referred to as configuration learning performance).

\section{Eyeblink startle paradigm}

In an eyeblink startle paradigm adapted from Jackson et al. (2003), participants viewed 30 reminder pictures from the distressing film fragments and 30 unrelated neutral (middle valence, low arousal ratings) pictures from the International Affective Picture System (IAPS; Lang, Bradley, \& Cuthbert, 2005). Additionally, 30 unrelated negative pictures (low valence, high arousal ratings) from the IAPS were included. This was done so as to contrast the expected modulation of startle responses to negatively valenced memories with a possible modulation of startle responses to acute negative affect induced by negative images. ${ }^{1}$ The reminder pictures consisted of screen captures from the trauma film fragments, such that the used scenes contained no graphically disturbing details (i.e., the reminder pictures were chosen to be as neutral as possible by themselves). Using Self-Assessment Manikin (Bradley \& Lang, 1999) ratings, we confirmed in a small independent sample $(N=15)$ that the reminder pictures were more neutral than the negative pictures in terms of both valence $\left(M_{\text {Reminder }}=4.32\right.$; $\left.M_{\text {Negative }}=2.75\right), t(14)=-10.29, p<.001$, and arousal $\left(M_{\text {Reminder }}=5.23 ; M_{\text {Negative }}=6.3\right), t(14)=4.74, p<.001$. Reminder pictures had slightly lower ratings of valence than did neutral IAPS pictures $\left(M_{\text {Neutral }}=5.21\right), t(14)=-8.16$, $p<.001$, as well as higher ratings of arousal $\left(M_{\text {Neutral }}=3.69\right)$, $t(14)=5.69, p<.001$.

Pictures were shown for $6 \mathrm{~s}$ (interstimulus interval $=14 \mathrm{~s}$ ), accompanied by a $50-\mathrm{ms}$ white noise startle probe $(95 \mathrm{~dB}$; near-instantaneous rise time) via headphones. Startle probe onset time was $2.5,4.5$, or $7 \mathrm{~s}$, relative to stimulus onset counterbalanced across stimulus categories, thus allowing the assessment of the chronometry of responding for each of the stimulus categories. In particular, the 2.5 - and 4.5 -s probes served to quantify affective responding, whereas the 7 -s probe served to assess the degree of affective down-regulation (Jackson et al., 2003). The trial sequence was randomized individually for each participant, with the restriction that no more than three consecutive trials had the same probe timing

\footnotetext{
${ }^{1}$ IAPS pictures used for the neutral control category were 2190, 2200, 2215, 2397, 2441, 2745.1, 5120, 5500, 5532, 5740, 7000, 7004, 7010, 7020, 7025, 7031, 7050, 7060, 7080, 7090, 7100, 7130, 7150, 7160, $7170,7175,8180,7224,7233,7500,7550,7700$; IAPS pictures used for the negative control category were 1052, 1070, 1090, 1120, 1220, $1280,1300,2120,2691,3000,3010,3016,3071,3100,3130,3150$, $3225,3261,3500,3530,6020,6190,6200,6230,6313,6370,6510$, 9040, 9254, 9410, 9423, 9490 .
}

or picture category. Six additional trials without startle probe were inserted into the trial sequence (two per picture category) in order to reduce the predictability of the startle probes.

Following the guidelines of Blumenthal et al. (2005), electromyography (EMG) was sampled continuously at $1000 \mathrm{~Hz}$, using $\mathrm{Ag} / \mathrm{AgCl}$ electrodes below the participants' left eye and an electrode on the forehead serving as signal ground. Electrode impedances were kept below $5 \mathrm{kOhms}$. EMG signals were rectified and $30 \mathrm{~Hz}$ low-pass filtered. For each trial, EMG signals were extracted from -50 to $250 \mathrm{~ms}$ relative to probe onset. The $50-\mathrm{ms}$ period before probe onset was used for baseline correction after rejection of noisecontaminated trials (i.e., signal changes $>20 \mu \mathrm{V}$ during baseline or reflex onset before probe onset). Data from 1 participant had to be excluded due to excessive artifacts. Reflex onset and peak values were extracted automatically between 20 and $120 \mathrm{~ms}$ after probe onset, yielding a startle magnitude per trial (peak minus onset values). Trials without eyeblink response were rated as zero. Startle magnitudes were square root transformed and averaged within subjects for each stimulus category and startle probe time. Startle potentiation (i.e., the relative strength of startle magnitude during reminder and unrelated negative trials, as compared with unrelated neutral trials) was statistically significant at the 4.5-s probe timing (for details, see Text 3, Supplementary Materials). For further analyses, startle potentiation scores were computed for reminder and unrelated negative trials per probe timing.

\section{Assessment of PTSD symptoms}

Intrusions were assessed using a 1-week diary (Holmes, James, Coode-Bate, \& Deeprose, 2009), with instructions to record intrusions as soon as they occurred. If no intrusions had occurred, participants were to record the absence of intrusions at least twice a day. The diary required participants to briefly describe each intrusion (for verification) and to indicate whether the intrusion was predominantly based on images, thoughts, or both. Intrusion frequencies were log-transformed prior to analyses to correct their right-skewed distribution. For each intrusion, participants also indicated how distressing it was on an 11point scale (anchors: $0=$ not at all; $10=$ extremely). Distress scores were averaged across all recorded intrusions. Intrusion-related distress was assessed additionally using the Intrusion Symptoms subscale of the Impact of Events Scale (IES; Horowitz, Wilner, \& Alvares, 1979) $(\alpha=$ .76) and the Re-Experiencing subscale of the self-report PTSD Symptom Scale (PSS-SR; Foa, Riggs, Dancu, \& Rothbaum, 1993) $(\alpha=.57)$. The total scores of both questionnaires were used to assess overall PTSD symptoms ( $\alpha=.83$ and .76, respectively). Both questionnaires were adapted to measure symptoms related to the trauma film. 


\section{Mood, anxiety, and depression}

The Positive and Negative Affect Schedule (PANAS; Watson, Clark, \& Tellegen, 1988) was used for repeated measurements of current positive affect (PA) and negative affect (NA) ( $\alpha \mathrm{s}>.74)$. The trait subscale of Spielberger's State-Trait Anxiety Inventory (STAI-T, Dutch translation; Van der Ploeg, 1985) was used to control for stable individual differences in anxiety levels $(\alpha=.89)$. Likewise, a Dutch version of the Beck Depression Inventory (Bouman, Luteijn, Albersnagel, \& Van der Ploeg, $1985)$ was used to control for general levels of depression $(\alpha=.87)$.

Procedure

Participants were invited to two laboratory sessions separated by a 1-week interval in which a diary was completed. During the first session, participants were seated in front of a computer screen at approximately $56 \mathrm{~cm}$ unrestrained viewing distance and completed a battery of computer-administered baseline questionnaires (not all questionnaire data are presented here), followed by the contextual cuing paradigm. Next and after preparing the skin with abrasive gel, the electrodes for the startle paradigm were attached. After that, the PANAS was administered, followed by a 14-min presentation of emotional video fragments. The stimulus materials largely overlapped with those used in Holmes et al. (2009), extended with similar video fragments. Participants were instructed to imagine being a witness to the scenes that consisted of explicit footage of genocide, medical surgeries, a drowning scene, and a car accident. During the trauma film presentation, participants were monitored via a closed-circuit video system to ensure adherence to the instructions. ${ }^{2}$ After the trauma film presentation, mood was reassessed with the PANAS. Following an unrelated and simple filler task (the Attention Network Test; Fan, McCandliss, Sommer, Raz, \& Posner, 2002) lasting $30 \mathrm{~min}$, participants underwent the eyeblink startle paradigm. At the end of the session, the electrodes were removed, and an appointment for a second session 1 week later was scheduled (with a tolerated deviation of 1 day). Finally, participants were given the diary and returned after 1 week to complete the selfreport questionnaire of PTSD symptoms.

\section{Statistical analysis}

To test our hypotheses, we employed Pearson product-moment correlations and multiple regressions, testing linear associations between independent and dependent variables. Repeated measures ANOVAs and $t$-tests were also used. When sphericity assumptions for ANOVAs were violated, Greenhouse-Geisser

\footnotetext{
$\overline{2}$ Two participants looked away for short periods of time during the film presentation but were not excluded from the sample.
}

corrected statistics are reported. ANOVAs are supplemented with partial eta squared $\left(\eta_{\mathrm{p}}{ }^{2}\right)$ and $t$-test with Cohen's $d$ as effect size estimates. Alpha was set at .05 (two-tailed) for all analyses.

\section{Results}

Effects of the trauma film

Affect changes due to the trauma film were assessed by comparing pre- and postfilm PANAS PA and NA scores using paired-samples $t$-tests. PANAS data from 1 participant were lost due to a database error in the electronic questionnaire system. Overall, PA decreased $\left(M_{\text {difference }}=-2.15\right.$, $S D=4.37), t(80)=-4.4, p<.001, d=-.39$, whereas NA increased $\left(M_{\text {difference }}=3.88, S D=4.60\right), t(80)=7.6$, $p<.001, d=.73$, following the trauma film. The mean scores of PTSD symptoms are summarized in Table 1.

Configuration learning performance (SCCT) and startle responses

Linear associations between SCCT learning and startle potentiation scores were tested independently for each type (reminder, unrelated negative) and probe timing $(2.5,4.5$, $7 \mathrm{~s})$. SCCT learning was unrelated to the two types of startle potentiation scores at the timings 2.5 and $4.5 \mathrm{~s}$ ( $r$ s ranging from -.08 to $.02, p s>.46)$. Neither was there an association with potentiation scores at the 7-s timing for reminder trials, $r=-.06$, n.s., whereas the correlation for negative trials approached significance, $r=-.202, p=.070$. In order to take all three timings and both types of startle potentiation into account, SCCT learning was entered as a two-level between-subjects factor (group allocation by median-split) in a 2 (group) $\times 2$ (type) $\times 3$ (timing) repeated measures ANOVA. No significant interaction of group by timing or by type ( $p s>.68$ ) was found, although the three-way interaction was borderline significant, $F(2,158)=2.66, p=.07$, $\eta_{\mathrm{p}}{ }^{2}=.03$. Despite the absence of statistical significance, we explored this interaction with post hoc paired-samples $t$-tests, which suggested that the group with poor learning on the SCCT $(n=40)$ showed larger startle potentiation during negative trials, as compared with reminder trials, at the 7-s timing, $t(39)=2.18, p=.035, d=.29$, whereas no such difference existed in the group with good SCCT learning $(n=41), t(40)=-0.32, p=.75, d=-.07$.

Configuration learning performance and PTSD symptoms

Direct linear associations between configuration learning performance and PTSD symptoms (i.e., intrusion frequency, distress, and overall symptoms) were assessed using correlation analyses. The results are summarized in Table 1. A 
Table 1 Pearson product-moment correlations between contextual learning performance and PTSD symptoms $(N=82)$

\begin{tabular}{llll}
\hline $\begin{array}{l}\text { PTSD symptoms } \\
\text { Intrusion frequency }\end{array}$ & & Sample mean $(S D)$ & Correlation \\
Method & Scale & & \\
Intrusion diary ${ }^{\text {a }}$ & Images & $2.76(3.16)$ & $-.33^{* *}$ \\
& Thoughts & $0.39(0.83)$ & .09 \\
& Images/thoughts & $1.06(1.77)$ & -.06 \\
& Sum & $4.21(4.15)$ & $-.28^{* *}$ \\
Intrusion distress & & & \\
Method & Scale & & -.12 \\
Intrusion diary & Distress & $3.50(2.04)$ & -.05 \\
IES & Intrusions & $6.02(5.24)$ & -.02 \\
PSS-SR & Reexperiencing & $3.34(1.81)$ & \\
Overall PTSD symptoms & & & -.03 \\
Method & Scale & & -.03 \\
IES & Total score & $9.95(8.56)$ & \\
PSS-SR & Total score & $6.30(4.69)$ &
\end{tabular}

Note. Contextual learning performance was calculated as the average median difference in reaction time between novel and repeated arrays across the first epoch of the spatial contextual cuing task. IES = Impact of Event Scale; PSS-SR = PTSD Symptom Scale-Self-Report.

${ }^{\mathrm{a}}$ The numbers of intrusions were log transformed prior to calculating correlation coefficients - that is, $\ln (1+\#$ intrusions $)$

** $p<.01$ (two-tailed)

significant negative correlation between SCCT learning and the total number of reported intrusions was found. This relationship was carried by intrusions that were perceptual (i.e., mental images), whereas SCCT learning was unrelated to the number of intrusions with a verbal component (i.e., thoughts or thought-image combinations). On the other hand, intrusion-related distress was unrelated to SCCT learning irrespective of assessment method, as were the total symptom scores on the PTSD scales.

Since one may hypothesize that other factors might be responsible for the effect of SCCT learning on the frequency of image-based intrusions, we tested this relationship while statistically correcting for trait anxiety, depression, and affective responding to the trauma film (i.e., PA and NA change scores). We therefore included the latter four factors in a multiple linear regression model (stepwise method; removal criterion: $p>.10$ ) and entered the factor SCCT learning subsequently. Only increases in NA due to the trauma film were kept as a significant predictor of perceptual intrusions, and entering SCCT learning significantly enhanced the model $\left(R^{2}\right.$ change $\left.=.10\right), F(1,78)=9.61, p<.01$. In this model, higher SCCT learning again predicted fewer image-based intrusions, $\beta=-.32, p<.01$, whereas stronger NA change predicted more image-based intrusions, $\beta=.27, p<.01$.

\section{Discussion}

The present study explored whether efficiency in learning visuospatial context information might be a relevant factor to explain individual differences in resilience after potentially traumatic experiences. The main findings of our study can be summarized as follows. We found that better configuration learning performance in a contextual cuing paradigm predicted significantly fewer intrusions during a 1-week period - specifically those intrusions that were purely perceptual in nature - over and above affective responding to the trauma film. In contrast, configuration learning performance was not predictive of intrusion-related distress and overall PTSD symptoms. Neither was there a direct relationship between learning performance in the contextual cuing paradigm and startle potentiation during or shortly after presentation of reminder pictures, aiming to activate memories of the trauma film. However, configuration learning performance tended to be negatively related to startle potentiation scores after the offset of unrelated negative pictures. Moreover, only in participants with relatively poor configuration learning performance, the latter potentiation scores appeared to be higher, as compared with potentiation scores of reminder pictures.

Efficiency of spatial configuration learning specifically reduces perceptual intrusions

Individuals with better configuration learning performance showed more resilience in terms of lowered levels of intrusions. This effect was specific for perceptual intrusions, possibly because they are generated differently than verbal intrusions. For instance, perceptual intrusions differ with respect to their level of abstraction and impact on emotion (Holmes \& Mathews, 2010), and it has been shown that the 
occurrence of intrusive thoughts can be manipulated independently of the occurrence of image-based intrusions (Hagenaars, Brewin, van Minnen, Holmes, \& Hoogduin, 2010)

Our study replicates and extends the findings of Bisby et al. (2010), who demonstrated a negative correlation between allocentric spatial recognition performance and perceptual intrusions in a trauma film paradigm. Differences between the contextual cuing paradigm employed in the present study and the recognition task used by Bisby and colleagues may be of relevance for theories of intrusions. They instructed participants to explicitly memorize spatial locations in a 3-D virtual space and to recognize configurations from a shifted viewpoint. This task requires a number of complex operations, including visualization, mental rotation, and explicit recognition, each of which may be (differently) related to the occurrence of intrusions. In contrast, in the contextual cuing paradigm, participants rely on repeated visual context information to aid search performance independently of, or even in the absence of, explicit recognition (Chun \& Phelps, 1999). Also, the contextual cuing effect may depend on a viewpoint-dependent rather than an allocentric spatial representation in memory (e.g., Chua \& Chun, 2003). Therefore, our data show that also less abstract and complex forms of visuospatial scene processing may be relevant in the prevention of perceptual intrusive experiences.

More speculatively, our findings can also be reconciled with the neuroanatomical predictions of the dualrepresentation model (Brewin et al., 2010), in which the hippocampus and parahippocampus play an essential role in the creation of coherent contextual memories and in the reduction of intrusive memories. The contextual cuing paradigm measures spatial memories that are processed implicitly in the parahippocampus (Preston \& Gabrieli, 2008), and more efficient spatial learning on this task would be expected to increase the input of spatial representations from the parahippocampus to the hippocampus (e.g., Fyhn et al., 2007). Therefore, our finding that higher learning efficiency in the contextual cuing paradigm is associated with fewer intrusions is in line with the view that memory formation in the hippocampal area helps to prevent intrusions, possibly by enabling the individual to form a coherent contextualized representation of a traumatic event.

Importantly, the present study failed to observe more global effects of contextual learning efficiency on clinically relevant outcomes such as distress and overall PTSD symptoms. This seems to contradict the previous findings by Gilbertson et al. (2007) showing a negative association between spatial configuration processing and overall symptom severity in PTSD patients. A number of reasons might account for the different findings. First, Gilbertson et al. assessed symptoms in PTSD patients, whereas we experimentally induced symptoms in individuals without prior psychopathology. It is therefore possible that visuospatial processing performance plays different roles in ongoing symptomatology and in the initial encoding process after viewing a distressing film. Second, and similar to the study of Bisby et al. (2010), the task used by Gilbertson et al. involves complex operations such as mental orientation and visualization, and these processes might be differently involved in the development and maintenance of PTSD symptoms than is learning performance in the contextual cuing paradigm. For instance, one might speculate that visualization requires efficient processing in parietal areas (Jordan et al., 2001), which have been proposed to play an important role in the interaction between sensation-based and contextbased memories in the dual-representation model (Brewin et al., 2010). Finally, Gilbertson et al. noted that their task was cognitively demanding, which introduces the possibility that their results may have been confounded by intelligence. Configuration learning in the SCCT occurs implicitly (i.e., with little cognitive demand) and can be readily distinguished from procedural learning (i.e., overall performance; Preston \& Gabrieli, 2008). At the same time, our sample consisted of relatively high-functioning individuals. Together, these two factors likely reduce potentially confounding effects of intelligence on task performance and symptom development. In sum, our and Gilbertson et al.'s findings suggest that visuospatial contextual encoding efficiency, likely reflecting activation in the parahippocampus, plays a crucial but specific role in the development of perceptual intrusions, whereas other mechanisms probably determine individual differences in the distress caused by intrusions (e.g., negative appraisals once an intrusion has occurred).

\section{Efficiency of spatial configuration learning and startle} responses

In order to explore whether configuration learning performance would be related to affective responding to trauma reminders, we used a startle paradigm, in which trauma film reminder pictures, as well as unrelated negative and neutral control pictures, were presented. We found no indication that configuration learning performance was linked to startle responses during or shortly after the presentation of reminder pictures. Also, we were unable to find a relationship between learning performance and the course of startle responding across the three startle timings, meaning that we failed to demonstrate the expected effect on the chronometry of affective responding to trauma film reminders. Thus, we found no support for the hypothesis that better configuration learning performance would lead to enhanced affective down-regulation (i.e., startle responding at the 7-s timing). This hypothesis was based on the assumption that SCCT performance is related to enhanced context-based memory formation, which would, in turn, be beneficial in 
the affect regulation controlled by the frontal cortex (Thayer et al., 2012).

One possible implication of our results is that affective processing of trauma-film-related memories may not be influenced by individual differences in the efficiency with which visuospatial context information is encoded. In terms of the dual-representation model (Brewin et al., 2010), our findings could suggest that the parahippocampal and amygdala-bound components in this model are not necessarily coupled. More generally, it is also possible that contextual embedding of a traumatic experience in memory does not necessarily imply that the affective response to the trauma cue will be dampened. Thus, other processes that have been suggested to play a role in the affective reaction to trauma memories may prove to be more important, including selective attention, negative appraisals, avoidance, and cognitive distortions (Brewin et al., 2010). However, since SCCT performance does not reflect contextual embedding of trauma memories directly, our data do not imply the absence of this link. Interestingly, the startle responses after unrelated negative pictures showed a trend toward affective hyperresponsivity in individuals with poorer contextual learning performance. This finding was unexpected, since configuration learning performance was expected to correlate with enhanced affect regulation only in response to affect-laden memories, but not during acute induction of negative affect. Although this trend-significant association was small and thus requires replication, one might speculate that this pattern of results indicates that the context-based memory system promotes contextual embedding when one is currently confronted with unrelated aversive stimuli, but not when reactivated trauma memories are processed.

Notably, the pattern of affective responding in our paradigm differed slightly from that in an earlier study with a similar method. That is, in Jackson et al. (2003), 47 participants displayed potentiated startle responses to negative images, irrespective of the timing at which the probe was presented. In contrast, we found that statistically significant startle potentiation during reminder and unrelated negative images occurred only at the $4.5 \mathrm{~s}$-timing, but not at 2.5 and $7 \mathrm{~s}$. Although it is unclear why these findings differ from previous ones, they are entirely in line with the expectation that affective responding would be strongest at $4.5 \mathrm{~s}$ (i.e., when the content of the picture has been fully apprehended), and weaker at $2.5 \mathrm{~s}$ (i.e., during initial visual processing) and $7 \mathrm{~s}$ (i.e., $1 \mathrm{~s}$ after picture offset, when emotion regulation occurs). Thus, both the reminder pictures and negative unrelated pictures evoked a heightened affective reaction, which was down-regulated after picture offset. Importantly, the startle potentiation at $4.5 \mathrm{~s}$ did not differ between the trials with reminder pictures and unrelated negative pictures, although the graphical content was more aversive in the latter pictures, which indicates that startle potentiation in the reminder pictures is likely caused by the activation of affectladen memories. The subsequent affective down-regulation apeared to be stronger for reminder pictures, since the startle magnitudes at $7 \mathrm{~s}$ were even lower than for neutral images, which was not the case for unrelated negative pictures. Thus, the pattern of findings suggests that we were able to adequately capture the time course of affective responding to trauma reminders, in order to investigate the relationship to individual differences in spatial configuration learning.

Assessing individual differences with the contextual cuing paradigm

To our knowledge, the present study is the first to use the contextual cuing paradigm (Chun \& Jian, 1998) in the context of individual-differences research. Using the SCCT (Bennett et al., 2009), we observed the typical contextual cuing effect, defined as reaction time advantage during visual search in repeated versus novel spatial configurations. Variation (i.e., individual differences) in this learning performance was strongest at the beginning of the task. In fact, individuals with low initial learning performance significantly improved in the course of the task, whereas individuals with high initial learning performance did not. Thus, even if individuals may differ little in learning performance in the final epoch of the task, there is considerable variation in the speed at which the learning effect is acquired. In other words, individual differences in the efficiency with which contextual information was learned apparently manifested themselves early rather than late during the task. We therefore decided to base our estimate of configuration learning performance on individual differences during the first ten repetitions of configurations (i.e., the first epoch). Moreover, learning performance was negatively associated with engagement in an active search strategy in the second epoch, but only in individuals with high initial learning performance. In line with this approach, Preston and Gabrieli (2008) showed that learning-related activation in the parahippocampus is strongest at the beginning of the task and declines after as few as two repetitions. This implies that in order to measure contextual learning efficiency, it may not be necessary to present a large number of visuospatial context repetitions.

\section{Limitations}

The following limitations deserve to be mentioned. To begin with, the present study was based on the assumption that spatial configuration learning would be related to the formation of context-based memories as hypothesized in the dual-representation model (Brewin et al., 2010). Although SCCT performance can be linked theoretically to the efficiency of the context-based memory system, we cannot exclude the possibility that the negative association between SCCT performance and intrusions was mediated by psychological processes other than the formation of context-based memories. That is, the present data cannot answer the 
question of whether individuals who are efficient in spatial configuration learning applied this efficiency to bind the features of the trauma film fragments. Therefore, future studies using a more direct assessment of contextualized memories next to configuration learning performance are needed. Also, the present study measured configuration learning performance only in relation to PTSD symptoms and involuntary memory (i.e., intrusions), but we did not assess explicit and voluntary memory performance. Therefore, no conclusions can be drawn about the specificity of SCCT effects on involuntary memory.

Another limitation is that the trauma film paradigm was used as an analogue condition to a traumatic event. It may be argued that trauma films are unable to elicit responses comparable to those of a potentially traumatic experience in real life. Also, we inserted a filler task (the Attention Network Test; Fan et al., 2002) to standardize the time between the trauma film and the subsequent startle paradigm. Although this task is very simple, it requires visuospatial attention, which may have had a dampening effect on the occurrence of intrusions (e.g., Holmes et al., 2009). Thus, a weak effect of the trauma film and, consequently, reduced statistical power may provide an alternative explanation for the absence of associations between configuration learning performance and the clinical and physiological parameters.

Another potential limitation is related to the use of reminder pictures to activate trauma memories in the startle paradigm. Since these images were selected to depict the low distressing aspects of the trauma film fragments, it is possible that full activation of the trauma film memories required a conscious retrieval process. Hypothetically, individuals with lower configuration learning performance in the SCCT may also have been marked by less efficient retrieval of the trauma film memories, which would have canceled out possible effects of configuration learning performance on startle responding. Although we cannot rule out this possibility, we found nevertheless that startle responses during full appraisal of neutral reminder pictures were significantly potentiated, as compared with unrelated neutral pictures, this potentiation being just as strong as that for unrelated negative pictures (see Text 3, Supplementary Materials). This suggests that the startle paradigm readily activated trauma-film-related memories and provided valid measurements of affective responding.

Finally, a potential threat to the external validity is related to our sample (i.e., undergraduates), which might be more skilled than the general population in emotion regulation and the adjustment to emotional provocation. Also, although we excluded participants who indicated high self-relevance of the trauma film (i.e., a history of specific traumatic experiences) and previous psychiatric problems on a self-report screening form, we did not systematically assess preexisting
PTSD symptoms, which may have influenced the results of this study.

Future considerations and conclusion

The present results have several implications for future research in the area of resilience and PTSD. First, while the present study demonstrated that more efficient spatial configuration learning contributes to individual differences in resilience in that it is related to lowered intrusion levels, the mechanism that mediates this effect remains obscure. Future studies may want to explore more directly the link between spatial configuration learning and the formation of context-based memories. For instance, the dual-representation model (Brewin et al., 2010) suggests that abstracted encoding of environmental patterns eventually helps to respond flexibly in novel situations and enhances higher cognitive functions, including narration and communication. Thus, one possible pathway from spatial configuration learning to the development of intrusions might be mediated by facilitated building of contextual memories, which would, in turn, lead to a better understanding of the stressful experience. However, it is also possible that the efficiency of spatial configuration learning is a mere epiphenomenon of intrusions, because individuals with more efficient parahippocampal-area-based configuration learning may also use the same brain area more efficiently for an unrelated basic psychological process, which may lead to suppression of intrusions. These issues remain to be addressed by future research.

Second, more efficient spatial configuration learning did not appear to inhibit affective processing of trauma reminders in terms of physiological responding, raising the question of whether the (parahippocampal component of the) context-based memory system contributes directly to affect regulation in reactivated trauma memories. Another interesting aspect is that there were (relatively weak) indications that individuals with more efficient configuration learning regulated their responses to unrelated negative pictures more efficiently. On the basis of this pattern of findings, one could speculate that more efficient spatial configuration learning indeed has a beneficial impact on the time course of affective responding, but only during the initial encoding of aversive perceptual information. Future studies might want to clarify this issue, next to investigating whether and how spatial configuration learning and context-based memory embedding contribute to resilience at later stages after a trauma-for instance, when trauma memories are retrieved and reconsolidated.

Third, both similarity and disparity of the present findings with previous studies into the role of spatial processing in PTSD (Bisby et al., 2010; Gilbertson et al., 2007) should inspire future studies to address important details of the theorized context-based memory system (Brewin et al., 
2010). For instance, while the specific effects on intrusion frequency reported here can be plausibly linked to implicit learning in the parahippocampus (Preston \& Gabrieli, 2008), it remains to be seen how such implicit learning processes might interact with more complex and explicit spatial processing (e.g., in the hippocampus) in the development of PTSD symptoms.

Finally, the finding that spatial configuration learning efficiency predicted perceptual intrusions, but not intrusion-related distress, may suggest that a combination of several factors accounts for the development of PTSD, each factor making rather specific contributions. Therefore, one could speculate that an inefficient processing in parts of the context-based memory system represents a nonspecific risk factor that generally increases the likelihood of experiencing more sudden recollections (not necessarily aversive ones; see Rubin, Boals, \& Berntsen, 2008). The actual development of PTSD after aversive experiences might thus depend additionally on other factors, such as the idiosyncratic interpretation of these intrusions and the cognitivebehavioral reaction to them (e.g., Steil \& Ehlers, 2000).

In conclusion, the present study demonstrates that the theoretical framework of the dual-representation model (Brewin et al., 2010) can be helpful in identifying and investigating a number of core mechanisms of resilience. Our results lend partial support to this model and suggest that efficiency in forming visuospatial context memories plays a specific role in image-based intrusive memories. Meanwhile, the distress related to intrusions and other PTSD symptoms appear to be unrelated to spatial configuration learning, prompting future research to investigate the role of contextbased memory formation in memory-related affect regulation. Thus, in order to understand resilience, it might be helpful to study the memory systems proposed by the dualrepresentation model in interaction with other factors such as cognitive-behavioral reactions to the occurrence of intrusions.

Acknowledgements This study was supported in part by Grant 05625-011 from the Netherlands Organization for Scientific Research (NWO) to Dr. Tom Smeets. The authors declare that no conflict of interest exists. We are especially thankful to Ron Hellenbrand, Jacco Ronner, and Felix Dücker (Maastricht University) for implementing the experimental paradigms and to Prof. Emily Holmes and Ella James (University of Oxford) for providing the materials for the trauma film paradigm, as well as to Dr. Ilana Bennett and her former colleagues from Georgetown University for providing their SCCT script and instructions that served as the basis for our study.

\section{References}

American Psychiatric Association. (2000). Diagnostic and Statistical Manual of Mental Disorders: DSM-IV, Text Revision. Washington, DC: Author.
Bennett, I. J., Barnes, K. A., Howard, J. H., \& Howard, D. V. (2009). An abbreviated implicit spatial context learning task that yields greater learning. Behavior Research Methods, 41, 391-395. doi:10.3758/brm.41.2.391

Bisby, J. A., King, J. A., Brewin, C. R., Burgess, N., \& Curran, H. V. (2010). Acute effects of alcohol on intrusive memory development and viewpoint dependence in spatial memory support a dual representation model. Biological Psychiatry, 68, 280-286.

Blumenthal, T. D., Cuthbert, B. N., Filion, D. L., Hackley, S., Lipp, O. V., \& Van Boxtel, A. (2005). Committee report: Guidelines for human startle eyeblink electromyographic studies. Psychophysiology, 42, 1-15. doi:10.1111/j.1469-8986.2005.00271.x

Bonanno, G. A. (2004). Loss, trauma, and human resilience - Have we underestimated the human capacity to thrive after extremely aversive events? American Psychologist, 59, 20-28. doi:10.1037/ 0003-066x.59.1.20

Bouman, T. K., Luteijn, F., Albersnagel, F. A., \& Van der Ploeg, F. A. G. (1985). Enige ervaringen met de Beck Depression Inventory (BDI) [Some experiences with the Beck Depression Inventory]. Gedrag - Tijdschrift voor Psychologie [Behavior - Journal of Psychology], 13, 13-24.

Bradley, M. M., \& Lang, P. J. (1999). Affective norms for English words (ANEW): Instruction manual and affective ratings. Gainesville, FL: Center for Research in Psychophysiology, University of Florida.

Brewin, C. R., Gregory, J. D., Lipton, M., \& Burgess, N. (2010). Intrusive images in psychological disorders: Characteristics, neural mechanisms, and treatment implications. Psychological Review, 117, 210-232. doi:10.1037/a0018113

Burgess, N., Maguire, E. A., \& O'Keefe, J. (2002). The human hippocampus and spatial and episodic memory. Neuron, 35, 625-641. doi:10.1016/s0896-6273(02)00830-9

Chua, K. P., \& Chun, M. M. (2003). Implicit scene learning is viewpoint dependent. Perception \& Psychophysics, 65, 72-80.

Chun, M. M., \& Jian, Y. H. (1998). Contextual cueing: Implicit learning and memory of visual context guides spatial attention. Cognitive Psychology, 36, 28-71.

Chun, M. M., \& Phelps, E. A. (1999). Memory deficits for implicit contextual information in amnesic subjects with hippocampal damage. Nature Neuroscience, 2, 844-847.

Davis, M., Walker, D. L., \& Lee, Y. L. (1997). Roles of the amygdala and bed nucleus of the stria terminalis in fear and anxiety measured with the acoustic startle reflex - Possible relevance to PTSD. Annals of the New York Academy of Sciences, 821, 305-331. doi:10.1111/j.1749-6632.1997.tb48289.x

de Quervain, D. J. F., Aerni, A., Schelling, G., \& Roozendaal, B. (2009). Glucocorticoids and the regulation of memory in health and disease. Frontiers in Neuroendocrinology, 30, 358-370. doi:10.1016/j.yfrne.2009.03.002

Delahanty, D. L., \& Nugent, N. R. (2006). Predicting PTSD prospectively based on prior trauma history and immediate biological responses. In R. Yehuda (Ed.), Psychobiology of Posttraumatic Stress Disorder: A Decade of Progress (Vol. 1071, pp. 27-40). Boston: New York Academy of Science.

Elzinga, B. M., \& Bremner, J. D. (2002). Are the neural substrates of memory the final common pathway in posttraumatic stress disorder (PTSD)? Journal of Affective Disorders, 70, 1-17.

Fan, J., McCandliss, B. D., Sommer, T., Raz, A., \& Posner, M. I. (2002). Testing the efficiency and independence of attentional networks. Journal of Cognitive Neuroscience, 14, 340-347. doi:10.1162/089892902317361886

Foa, E. B., Riggs, D. S., Dancu, C. V., \& Rothbaum, B. O. (1993). Reliability and validity of a brief instrument for assessing posttraumatic stress disorder. Journal of Traumatic Stress, 6, 459473.

Francati, V., Vermetten, E., \& Bremner, J. D. (2007). Functional neuroimaging studies in posttraumatic stress disorder: Review of 
current methods and findings. Depression and Anxiety, 24, 202218. doi:10.1002/da.20208

Fyhn, M., Hafting, T., Treves, A., Moser, M. B., \& Moser, E. I. (2007). Hippocampal remapping and grid realignment in entorhinal cortex. Nature, 446, 190-194.

Fyhn, M., Molden, S., Witter, M. P., Moser, E. I., \& Moser, M. B. (2004). Spatial representation in the entorhinal cortex. Science, 305, 1258-1264. doi:10.1126/science.1099901

Gilbertson, M. W., Williston, S. K., Paulus, L. A., Lasko, N. B., Gurvits, T. V., Shenton, M. E., et al. (2007). Configural cue performance in identical twins discordant for posttraumatic stress disorder: Theoretical implications for the role of hippocampal function. Biological Psychiatry, 62, 513-520. doi:10.1016/ j.biopsych.2006.12.023

Hagenaars, M. A., Brewin, C. R., van Minnen, A., Holmes, E. A., \& Hoogduin, K. A. L. (2010). Intrusive images and intrusive thoughts as different phenomena: Two experimental studies. Memory, 18, 76-84. doi:10.1080/09658210903476522

Holmes, E. A., Brewin, C. R., \& Hennessy, R. G. (2004). Trauma films, information processing, and intrusive memory development. Journal of Experimental Psychology. General, 133, 3-22. doi:10.1037/0096-3445.133.1.3

Holmes, E. A., James, E. L., Coode-Bate, T., \& Deeprose, C. (2009). Can playing the computer game "Tetris" reduce the build-up of flashbacks for trauma? A proposal from cognitive science. PLoS One, 4, 6. doi:e415310.1371/journal.pone.0004153

Holmes, E. A., \& Mathews, A. (2010). Mental imagery in emotion and emotional disorders. Clinical Psychology Review, 30, 349-362. doi:10.1016/j.cpr.2010.01.001

Horowitz, M. J., Wilner, N., \& Alvares, W. (1979). Impact of Event Scale: A measure of subjective stress. Psychosomatic Medicine, 41, 209-218.

Jackson, D. C., Mueller, C. J., Dolski, I., Dalton, K. M., Nitschke, J. B., Urry, H. L., et al. (2003). Now you feel it, now you don't: Frontal brain electrical asymmetry and individual differences in emotion regulation. Psychological Science, 14, 612-617.

Jordan, K., Heinze, H. J., Lutz, K., Kanowski, M., \& Jancke, L. (2001). Cortical activations during the mental rotation of different visual objects. NeuroImage, 13, 143-152.

Lang, P. J., Bradley, M. M., \& Cuthbert, B. N. (2005). International Affective Picture System (IAPS): Instruction Manual and Affective Ratings. Technical Report A-6. Gainesville, FL: University of Florida.

Lleras, A., \& Von Muhlenen, A. (2004). Spatial context and top-down strategies in visual search. Spatial Vision, 17, 465-482.

Manns, J. R., \& Squire, L. R. (2001). Perceptual learning, awareness, and the hippocampus. Hippocampus, 11, 776-782. doi:10.1002/hipo.1093

Marmar, C. R., McCaslin, S. E., Metzler, T. J., Best, S., Weiss, D. S., Fagan, J., et al. (2006). Predictors of posttraumatic stress in police and other first responders. In R. Yehuda (Ed.), Psychobiology of posttraumatic stress disorder: A decade of progress (pp. 1-18). Boston: New York Academy of Science.

Murray, E. A., Bussey, T. J., \& Saksida, L. M. (2007). Visual perception and memory: A new view of medial temporal lobe function in primates and rodents. Annual Review of Neuroscience, 30, 99122. doi:10.1146/annurev.neuro.29.051605.113046

Orr, S. P., \& Roth, W. T. (2000). Psychophysiological assessment: Clinical applications for PTSD. Journal of Affective Disorders, 61, 225-240.

Ozer, E. J., Best, S. R., Lipsey, T. L., \& Weiss, D. S. (2003). Predictors of posttraumatic stress disorder and symptoms in adults: A metaanalysis. Psychological Bulletin, 129, 52-73. doi:10.1037//00332909.129.1.52

Preston, A. R., \& Gabrieli, J. D. E. (2008). Dissociation between explicit memory and configural memory in the human medial temporal lobe. Cerebral Cortex, 18, 2192-2207. doi:10.1093/ cercor/bhm 245

Rubin, D. C., Berntsen, D., \& Bohni, M. K. (2008a). Memory-based model of Posttraumatic Stress Disorder: Evaluating basic assumptions underlying the PTSD diagnosis. Psychological Review, 115, 985-1011. doi:10.1037/a0013397

Rubin, D. C., Boals, A., \& Berntsen, D. (2008b). Memory in Posttraumatic Stress Disorder: Properties of voluntary and involuntary, traumatic and nontraumatic autobiographical memories in people with and without Posttraumatic Stress Disorder symptoms. Journal of Experimental Psychology. General, 137, 591-614. doi:10.1037/a0013165

Shin, L. M., Rauch, S. L., \& Pitman, R. K. (2006). Amygdala, medial prefrontal cortex, and hippocampal function in PTSD. In R. Yehuda (Ed.), Psychobiology of Posttraumatic Stress Disorder: A decade of progress (Vol. 1071, pp. 67-79). Boston: New York Academy of Science.

Steil, R., \& Ehlers, A. (2000). Dysfunctional meaning of posttraumatic intrusions in chronic PTSD. Behaviour Research and Therapy, 38, 537-558. doi:10.1016/s0005-7967(99)00069-8

Suvak, M. K., \& Barrett, L. F. (2011). Considering PTSD from the perspective of brain processes: A psychological construction approach. Journal of Traumatic Stress, 24, 3-24. doi:10.1002/ jts. 20618

Thayer, J. F., Ahs, F., Fredrikson, M., Sollers, J. J., \& Wager, T. D. (2012). A meta-analysis of heart rate variability and neuroimaging studies: Implications for heart rate variability as a marker of stress and health. Neuroscience and Biobehavioral Reviews, 36, 747756. doi:10.1016/j.neubiorev.2011.11.009

Van der Ploeg, H. M. (1985). The development and validation of the Dutch State-Trait Anxiety Inventory. In C. D. Spielberger \& I. G. Sarason (Eds.), Stress and Anxiety (pp. 129-139). Washington, DC: Hemisphere.

van Strien, N. M., Cappaert, N. L. M., \& Witter, M. P. (2009). The anatomy of memory: An interactive overview of the parahippocampal-hippocampal network. Nature Reviews Neuroscience, 10, 272-282. doi:10.1038/nrn2614

Watson, D., Clark, L. A., \& Tellegen, A. (1988). Development and validation of brief measures of positive and negative affect: The PANAS scales. Journal of Personality and Social Psychology, 54, 1063-1070. 